\title{
Expression of Spindle and Kinetochore-related Complex Subunit 3 is Associate With the Progression and Prognosis of Hepatocellular Carcinoma and Pancreatic Adenocarcinoma
}

Kuiwei Su

Bengbu Medical College

Yu Huang

Zhejiang Chinese Medical University

Shufang Xie

Zhejiang Chinese Medical University

Chen Yang

Qingdao University

Guoqing Ru

Zhejiang Provincial People's Hospital

Xianglei He

Zhejiang Provincial People's Hospital

Wanyuan Chen

Zhejiang Provincial People's Hospital

Hongying Pan

Zhejiang Provincial People's Hospital

Gang Cheng

Zhejiang Provincial People's Hospital

Shibing Wang ( $\nabla$ wangshibing@hmc.edu.cn)

Zhejiang Provincial People's Hospital

Research article

Keywords: SKA3, biomarker, hepatocellular carcinoma, pancreatic adenocarcinoma, Bioinformatics

Posted Date: July 10th, 2020

DOI: https://doi.org/10.21203/rs.3.rs-40794/v1

License: (c) (1) This work is licensed under a Creative Commons Attribution 4.0 International License.

Read Full License 
Page $2 / 21$ 


\section{Abstract \\ Background}

Several studies have shown that Spindle and Kinetochore-related Complex Subunit 3 (SKA3) is closely related to the diagnosis and prognosis of various cancers. SKA3 plays a vital role in mitosis, but the mechanism underlying its action in tumors has not been fully elucidated.

\section{Methods}

Two separate microarrays of pancreatic adenocarcinoma (PAAD) and hepatocellular carcinoma (HCC) samples were downloaded from the Gene Expression Omnibus (GEO) and analyzed using R software. Tissues from 203 HCC and 321 PAAD patients were used for tissue microarray (TMA) construction and immunohistochemical staining for scoring. Kaplan-Meier estimators were used to determine their clinical relevance. Finally, Gene ontology (GO) and Kyoto Encyclopedia of Genes and Genomics (KEGG) were used for analyzed rich pathways of SKA3 to understand gene function further.

\section{Results}

Contrary to the results obtained from the GEO database, HCC and PAAD tumor tissues exhibited significantly lower expression of SKA3 compared to the adjacent tissues $(P<0.001)$. The size of HCC tumor and Edmondson grade were statistically correlated with SKA3 expression $(P<0.05)$. No correlation was observed between the levels of SKA3 expression and vascular invasion, metastasis, or lymphatic infiltration in HCC patients. Moreover, factors related to decreases of SKA3 in PAAD could similarly not be found. Kaplan-Meier survival curves indicated that SKA3 upregulation in HCC was significantly associated with better prognosis $(P=0.016)$. In contrast, low levels of SKA3 were related to poor prognosis of PAAD patients $(P=0.026)$. Enrichment analysis of $G O$ and KEGG pathways showed that SKA3 gene was closely related to cell cycle pathways.

\section{Conclusions}

The results of the present study indicate that SKA3 expression can serve as a diagnostic and prognostic biomarker for HCC and PAAD. However, there is a need to conduct further studies to verify these findings, especially the prognostic role of SKA3 in PAAD.

\section{Background}

Spindle and kinetochore-related complex subunit 3 (SKA3), also referred to as C13orf3, is a vital component of spindle and kinetochore-related complexes with a predicted molecular weight of $45 \mathrm{kDa}$ [1]. SKA3 controls the separation of chromosomes in mitosis. As such, its depletion leads to a shutdown of 
the mitotic process. Also, it can delay the late stage of mitosis and facilitate the accumulation of the anaphase-promoting complex/cyclosome body (APC/C) on the chromosome, thereby slowing down the mitotic cycle [2].

Recent studies have implicated SKA3 expression in the prognosis of several cancers [3-5]; however, its role in the progression of pancreatic adenocarcinoma (PAAD) and hepatocellular carcinoma (HCC) remains unclear. Herein, we aimed to examine the correlation between SKA3 and HCC and PAAD.

PAAD is a highly invasive malignancy and one of the deadliest cancers among solid tumors. The disease has a very poor prognosis with a five-year survival rate of less than $10 \%$. Currently, its case fatality rate exceeds $95 \%$ [6]. At present, PAAD can only be treated with surgery. However, due to the lack of specific symptoms at its formative stage and the limitations of existing diagnostic methods, most patients are diagnosed as advanced and often have accompanying metastasis, resulting in a failure to undergo radical surgical excision [7]. Despite its poor prognosis, effective treatment options for PAAD are still lacking. Numerous potential biomarkers have been identified, including b7-h4, MUC13. However, none has been able to precisely predict the presence and outcomes of the disease, which is crucial in guiding the course of treatment of PAAD patients $[8,9]$.

HCC is among the five most prevalent cancers globally. As such, it claims numerous lives worldwide each year [10]. Despite the development of innovative therapies, such as liver transplantation, liver resection, immunotherapy, and chemotherapy [11], the prognosis of HCC is often poor due to its high recurrence and metastasis rates $[12,13]$. Many biomarkers have been identified to predict the prognosis and guide HCC treatment. These include Coxsackie and adenovirus receptors (CAR) [14]DICER1,GMPSINCOR1 [15]and PD-L1 [16]. However, these biomarkers have not been effective in the diagnosis and prognosis of the disease. Given this, there is a need to look for better biomarkers.

Here, we compared the expression level of SKA3 in PAAD or HCC tissue samples versus their adjacent tissue samples. In addition, we examined the associations between clinical parameters and SKA3 expression to establish the prognostic value of SKA3 in HCC and PAAD patients.

\section{Materials And Methods}

\section{Gene expression profiling interactive analysis}

The gene expression profiling interactive analysis (GEPIA) (http://gepia.cancer-pku.cn) database [17] was used to identify the patients with HCC and PAAD mRNA expression and its relationship with prognosis.

\section{Microarray datasets}

Gene Expression Omnibus (GEO, http://www.ncbi.nlm.nih.gov/geo) is a public and open database [18]. In this study, two independent HCC(GSE62044) and PAAD(GSE28735) microarrays were downloaded from the GEO to explore SKA3 expression. Subsequently, The SKA3 H-score median was then used to divide the samples into the high-expression group and the low-expression group. Next, we used the adjusted 
$|\log 2 \mathrm{FC}|>1.5$ and $\mathrm{p}$-value $<0.05$ as truncation criteria to identify the differentially expressed genes (DEGs) between the two groups of samples. Finally, Gene Ontology (GO) and Kyoto Encyclopedia of Genes and Genomes (KEGG) pathway enrichment analyses were performed to identify DEGs.

\section{The human protein atlas}

Human protein atlas (https://www.proteinatlas.org) was used to assess the expression of SKA3 protein in $\mathrm{HCC}$ and PAAD tissues versus their matching normal tissues. The project contains immunohistochemical $(\mathrm{IHC})$ data derived from TMA-based analysis of different types of normal and cancer tissues [19]. The number of patients with various types of cancer, clinical information, and staining intensity are provided online.

\section{Tissue specimens and cancer patients}

The data of HCC or PAAD patients who underwent radical resection between 2008-2015 at Zhejiang Provincial People's Hospital were collected and used to construct tissue microarray (TMA). In total, 321 HCC patients comprising 261 males and 60 females with a median age of 57 years (25-90) were included. Also included were 203 PAAD patients consisting of 132 males and 71 females, with a median age of 63 years (30-91). The period starting from the surgical operation date to the end of follow-up (December 2016) or the date of death was used to calculate survival time. This study was approved by the Ethics Committee of the Zhejiang Provincial People's Hospital. All patients provided written informed consent before conducting any study-related procedures.

\section{Immunohistochemical staining}

Immunohistochemistry (IHC) was performed as described previously [20]. Briefly, we baked tissue microarrays in $5 \mu \mathrm{m}$ sections for two hours at $70^{\circ} \mathrm{C}$. The tissues were removed from paraffin using xylene, the rehydrated using a descending ethanol concentration gradient. Antigen retrieval was performed through boiling for 3 minutes in a high-pressure cooker filled with Tris-EDTA (1 mM) buffer (HuaBio, Hangzhou, China). Endogenous peroxidases were blocked for $15 \mathrm{~min}$ using hydrogen peroxide (3\%, Sinopharm Chemical Reagent Company, China) then treated with bovine serum albumin (BSA) [1\%, Sigma, United States] for 20 minutes to reduce unspecific staining. Next, the sections were probed with a 1:50 dilution of anti-SKA3 antibody (rabbit polyclonal; Abcam, Cambridge, United Kingdom) overnight at $4{ }^{\circ} \mathrm{C}$ followed by a secondary antibody immunohistochemical kit (MXB, Fuzhou, China) for half an hour at room temperature. A diaminobenzidine (DAB) reaction Kit (Gene Tech, Shanghai, China) was used to generate staining localized to sites where the secondary antibody was stably bound. The sections were subjected to a final hematoxylin and eosin (HE) counterstaining, dehydration, and clearing before they were mounted on slides for microscopic examination.

\section{Assessment of IHC stained tissue sections}

We set the dark blue on the tissue section as strong positive, sky blue as moderate positive, light blue as weak positive, and unstained as negative. Then we used the Densito Quant software in Quant Center to automatically identify, and finally get the $\mathrm{H}$-score (histochemistry score). The median of the H-score of 
the two tumors was selected to measure the expression level of SKA3. An expression greater than or equal to the median was regarded as high, whereas an expression less than the median was taken as low. The following were the thresholds used to define levels of SKA3 expression in HCC samples: low were scores $<164$ and high were scores $>=64$. For PAAD samples, the thresholds were: low-scores $<148$ and high-scores $>=148$.

\section{GO and KEGG pathway enrichment analysis}

Gene ontology (G0) [http://www.geneontology.org/] is a common bioinformatics tool. The Kyoto Encyclopedia of Genes and Genomics (KEGG http://www.genome.jp/kegg/) is an online database for analyzing rich pathways of selected genes to understand gene function further. $P<0.05$ was considered statistically significant.

\section{Statistical analysis}

Data analysis was performed using the Statistical Package for Social Sciences (version 22.0; SPSS Inc., Illinois, United States). Univariate and multivariate factors independently apportioning risk were evaluated using the Cox proportional hazards model. Statistical significance was set at $p<0.05$.

\section{Results}

This study was divided into three parts. In the first part, we compared the SKA3 mRNA expression levels in HCC and PAAD tissues using the GEPIA database to investigate the relationship between SKA3 protein expression and HCC or PAAD prognosis. In the second part, SKA3 protein expression in tissue microarray (TMA) was evaluated and compared with the database. Finally, we filtered out the differentially expressed genes (DEG) of SKA3 and made corresponding functional comments.

\section{mRNA levels of SKA3 in patients with HCC and PAAD}

The expression and transcription levels of SKA3 in HCC and PAAD tissues were compared with those in corresponding control tissues (normal tissues) in the GEPIA data set. In total, $369 \mathrm{HCC}$ versus 160 normal tissues (Fig. 1A) and 179 PAAD versus 171 normal tissues (Fig. 1B) from the GEPIA database were analyzed. Based on the results, the levels of SKA3 mRNA in HCC and PAAD tissues were higher than those in normal tissues. Subsequently, we investigated the protein profile of humans to further analyze the expression at the protein level. The immunohistochemical results of Human Protein Atlas showed that SKA3 staining was not detected in normal liver and pancreas tissues (Fig. 1C, D). Collectively, these data indicated that SKA3 was up-regulated at the transcription level but its expression level in the proteome remains unclear.

\section{Relationship between SKA3 mRNA expression and stage or prognosis of HCC patients}


Based on GEPIA analysis, the expression of SKA3 mRNA in HCC and PAAD samples was not significantly different from that in the advanced clinical stage (Fig. 1E, F; P > 0.01). Subsequently, survival analysis using GEPIA showed that higher SKA3 expression was significantly correlated with shorter OS in patients with HCC or PAAD (Fig. 1G, H; P< 0.05). Higher levels of SKA3 mRNA was closely associated with poor clinical prognosis of both liver and pancreatic cancers.

\section{SKA3 protein expression in PAAD or HCC tissues versus their marched normal tissues}

We evaluated the expression level of SKA3 protein in TMA to verify further the results obtained from the bioinformatics database. IHC staining was used to compare the levels of SKA3 protein between tumor tissues and their matched normal tissues obtained from 80 PAAD and $128 \mathrm{HCC}$ patients (Fig. 2A, B, C, D). Comparison of SKA3 expression levels between the cancer tissues and their matched normal tissues with Mann-Whitney $\mathrm{U}$ test revealed significant reductions in SKA3 protein expression in cancer tissues of both diseases $(P<0.05)$ [figure $2 E, F]$.

\section{The association between SKA3 expression and clinical characteristics of HCC or PAAD}

Here, we examined the relationship between clinical characteristics of 203 PAAD or 321 HCC patients and SKA3 levels. The patients were divided into either high or low SKA3 groups based on IHC tumor staining. Expression levels of SKA3 in HCC patients were positively correlated with tumor size and Edmondson grade $(P<0.05$, Table 1$)$. However, this was not replicated in any of the other clinical characteristics, including tumor number, age, gender, alpha-fetoprotein (AFP) level, metastasis, vessel invasion, HBs antigen, cirrhosis, and microvascular invasion (Table 1). Interestingly, we did not find any factors associated with a decrease in SKA3 in PAAD patients, suggesting that SKA3 might be a potential indicator of disease progression in HCC tumors. 
Table 1

Clinical characteristics of hepatocellular carcinoma patients correlate with SKA3 expression

\begin{tabular}{|c|c|c|c|c|}
\hline \multirow[t]{2}{*}{ Clinical parameters } & \multirow[t]{2}{*}{ Total } & \multicolumn{2}{|c|}{ SKA3 expression } & \multirow[t]{2}{*}{$P$ value } \\
\hline & & Low & high & \\
\hline Age (years) & & & & 0.076 \\
\hline$<55$ & 116 & 65 & 51 & \\
\hline$\geq 55$ & 193 & 88 & 105 & \\
\hline Gender & & & & 0.948 \\
\hline Male & 252 & 125 & 127 & \\
\hline Female & 57 & 28 & 29 & \\
\hline Size(cm) & & & & $0.014^{*}$ \\
\hline$<=5$ & 179 & 79 & 100 & \\
\hline$>5$ & 123 & 72 & 51 & \\
\hline Tumor number & & & & 0.262 \\
\hline Single & 254 & 122 & 132 & \\
\hline Multiple & 55 & 31 & 24 & \\
\hline Edmondson grade & & & & $0.046^{*}$ \\
\hline I-II & 195 & 105 & 90 & \\
\hline III-IV & 114 & 48 & 66 & \\
\hline Metastasis & & & & 0.862 \\
\hline MO & 279 & 140 & 139 & \\
\hline M1 & 25 & 13 & 12 & \\
\hline microvascular invasion & & & & 0.150 \\
\hline Absence & 116 & 52 & 64 & \\
\hline Presence & 118 & 64 & 54 & \\
\hline \multicolumn{5}{|c|}{ American Joint Committee on Cancer Staging (2010). } \\
\hline \multicolumn{5}{|l|}{ HBV: hepatitis B virus. } \\
\hline \multicolumn{5}{|l|}{ AFP: alpha-fetoprotein. } \\
\hline${ }^{*} \mathrm{P}<0.05$, statistically & $P$ & & & \\
\hline
\end{tabular}




\begin{tabular}{|c|c|c|c|c|}
\hline \multirow[t]{2}{*}{ Clinical parameters } & \multirow[t]{2}{*}{ Total } & \multicolumn{2}{|c|}{ SKA3 expression } & \multirow[t]{2}{*}{$P$ value } \\
\hline & & Low & high & \\
\hline \multicolumn{4}{|l|}{ HBV } & 0.610 \\
\hline Absence & 56 & 26 & 30 & \\
\hline Presence & 247 & 124 & 123 & \\
\hline \multicolumn{4}{|l|}{ Cirrhosis } & 0.467 \\
\hline Absence & 99 & 52 & 47 & \\
\hline Presence & 210 & 101 & 109 & \\
\hline \multicolumn{4}{|l|}{ AFP levels } & 0.147 \\
\hline$<=400 \mu \mathrm{g} / \mathrm{L}$ & 184 & 87 & 97 & \\
\hline$>400 \mu \mathrm{g} / \mathrm{L}$ & 64 & 37 & 27 & \\
\hline \multicolumn{5}{|c|}{ American Joint Committee on Cancer Staging (2010). } \\
\hline \multicolumn{5}{|l|}{ HBV: hepatitis B virus. } \\
\hline \multicolumn{5}{|c|}{ AFP: alpha-fetoprotein. } \\
\hline \multicolumn{5}{|c|}{${ }^{*} \mathrm{P}<0.05$, statistically significant, Pearson's $\chi^{2}$ test was applied to analyze. } \\
\hline
\end{tabular}


Table 2

Clinical characteristics of pancreatic cancer patients correlate with SKA3 expression

\begin{tabular}{|c|c|c|c|c|}
\hline \multirow[t]{2}{*}{ Clinical parameters } & \multirow[t]{2}{*}{ Total } & \multicolumn{2}{|c|}{ SKA3 expression } & \multirow[t]{2}{*}{$P$ value } \\
\hline & & Low & high & \\
\hline Gender & & & & 0.460 \\
\hline Male & 129 & 67 & 62 & \\
\hline Female & 71 & 33 & 38 & \\
\hline Age(years) & & & & 0.471 \\
\hline$<55$ & 38 & 21 & 17 & \\
\hline$>=55$ & 162 & 79 & 83 & \\
\hline Size(cm) & & & & 0.347 \\
\hline$<=5$ & 51 & 23 & 28 & \\
\hline$>5$ & 146 & 77 & 69 & \\
\hline Tumor cell differentiation & & & & 1.000 \\
\hline None & 10 & 5 & 5 & \\
\hline Poorly & 92 & 46 & 46 & \\
\hline Moderately & 85 & 43 & 42 & \\
\hline High & 4 & 2 & 2 & \\
\hline Metastasis & & & & 0.858 \\
\hline M0 & 123 & 64 & 59 & \\
\hline M1 & 71 & 36 & 35 & \\
\hline Vessel invasion & & & & 0.295 \\
\hline Absence & 114 & 60 & 54 & \\
\hline Presence & 46 & 20 & 26 & \\
\hline Distant metastases & & & & 0.093 \\
\hline Negative & 67 & 37 & 30 & \\
\hline Positive & 46 & 18 & 28 & \\
\hline
\end{tabular}

American Joint Committee on Cancer Staging (2010).

* $P<0.05$, statistically significant, Pearson's $\chi^{2}$ test was applied to analyze. 


\begin{tabular}{|c|c|c|c|c|}
\hline \multirow[t]{2}{*}{ Clinical parameters } & \multirow[t]{2}{*}{ Total } & \multicolumn{2}{|c|}{ SKA3 expression } & \multirow[t]{2}{*}{$P$ value } \\
\hline & & Low & high & \\
\hline \multicolumn{4}{|l|}{ lymphatic vessel invasion } & 0.380 \\
\hline Negative & 44 & 20 & 24 & \\
\hline Positive & 18 & 6 & 12 & \\
\hline \multicolumn{5}{|c|}{ American Joint Committee on Cancer Staging (2010). } \\
\hline \multicolumn{5}{|c|}{ * $P<0.05$, statistically significant, Pearson's $\chi^{2}$ test was applied to analyze. } \\
\hline
\end{tabular}

According to Kaplan-Meier curves, high expression of SKA3 in cancerous tissues conferred HCC patients with longer overall survival (OS) time compared to patients with low SKA3 levels ( $P=0.016$, Fig. $2 G$ ). Patients with low SKA3 expression had a median survival time of 31 months. The median survival time of patients with high expression of SKA3 was 61 months.

In HCC, Cox regression analysis indicated that low SKA3 expression was an independent prognostic factor for shorter OS time (Table 3). We also examined the prognostic values of other conceivable risk factors including, tumor size, grade, microvascular penetration, vessel invasion, metastasis, and AFP in predicting OS of HCC patients. Notably, Cox multivariate analysis revealed that SKA3 expression, metastasis, and Edmondson grade had a more significant effect on prognosis.

Table 3

Assessment of clinicopathological parameters of hepatocellular carcinoma patients and their multivariate or univariate association with SKA3 expression using Cox regression.

\begin{tabular}{|llllll|}
\hline Parameters & \multicolumn{2}{l}{ Univariate analyses } & & & \multicolumn{2}{c|}{ Multivariate analyses } & \\
\cline { 2 - 3 } \cline { 6 - 6 } & Hazard ratio $(95 \% \mathrm{Cl})$ & P value & & Hazard ratio (95\%Cl) & P-value \\
\hline SKA3 expression & $0.375-0.964$ & 0.035 & & $0.102-0.577$ & 0.001 \\
\hline Size & $1.230-3.236$ & 0.005 & & $0.333-1.846$ & 0.577 \\
\hline Metastasis & $2.603-9.335$ & 0.000 & & $2.310-23.100$ & 0.001 \\
\hline Microvascular invasion & $1.137-3.291$ & 0.015 & & $0.270-9.236$ & 0.613 \\
\hline Vessel invasion & $1.325-3.972$ & 0.002 & & $0.160-5.760$ & 0.965 \\
\hline Edmondson grade & $1.768-4.651$ & 0.000 & & $1.498-8258$ & 0.004 \\
\hline AFP & $1.401-4.705$ & 0.002 & & $0.730-3.662$ & 0.232 \\
\hline Cl, confidence interval. & & & & \\
\hline
\end{tabular}


In contrast, Kaplan-Meier curves revealed that PAAD patients with low SKA3 expression had longer OS time than those with high SKA3 expression $(P=0.026$, Fig. $2 \mathrm{H})$. Patients with low SKA3 expression had an average overall survival time of 22 months, whereas 12.5 months was the median OS time of patients exhibiting high SKA3 expression. It is noteworthy that among all other clinical features, only tumor size had a significant effect on the prognosis of PAAD patients, as revealed by COX regression analysis (Table 4).

Table 4

Assessment of clinicopathological parameters of pancreatic cancer patients and their multivariate or univariate association with SKA3 expression using Cox regression.

\begin{tabular}{|c|c|c|c|c|}
\hline \multirow[t]{2}{*}{ Parameters } & \multicolumn{2}{|l|}{ Univariate analyses } & \multicolumn{2}{|l|}{ Multivariate analyses } \\
\hline & Hazard ratio $(95 \% \mathrm{Cl})$ & $P$ value & Hazard ratio (95\%Cl) & P-value \\
\hline SKA3 expression & $0.729-1.731$ & 0.597 & $0.282-3.142$ & 0.921 \\
\hline Size & $1.010-3.396$ & 0.046 & $0.172-2.558$ & 0.552 \\
\hline Gender & $0.443-1.069$ & 0.097 & $0.298-2.472$ & 0.776 \\
\hline Location & $0.807-1.119$ & 0.540 & $0.702-1.661$ & 0.727 \\
\hline Cancer metastasis & $0.924-2.124$ & 0.113 & $0.257-3.462$ & 0.929 \\
\hline Differentiation & $0.513-1.008$ & 0.056 & $0.248-1.661$ & 0.361 \\
\hline Lymphatic vessel invasion & $0.832-4.064$ & 0.132 & $0.450-6.827$ & 0.419 \\
\hline $\mathrm{Cl}$, confidence interval. & & & & \\
\hline
\end{tabular}

We performed further analysis to study the biological roles of SKA3 in HCC and PAAD. To screen for differential genes associated with SKA3, according to the median value of SKA3 expression,the genes in the HCC (GSE62044) and PAAD (GSE28735) data sets were divided into the low and high SKA3 expression groups. A total of 114 up-regulated and 8 downregulated genes were identified in HCC (Fig. 3A). On the other hand, 196 up-regulated and 95 down-regulated genes were identified in PAAD (Fig. 3B). The heat maps showed the top 20 important DEGs with positive and negative correlations (Figs. 3C, D). Subsequently, GO annotation and KEGG pathway enrichment analyses were performed on the DEG of the first 20 genes. In the HCC and PAAD organizations,

the genes with the highest gene enrichment are closely related to nuclear division, organelles fission, chromosome segregation, mitotic nuclear division and nuclear chromosome separation (Fig. 4A, B). Notably, KEGG analysis showed that DEG overlapping in HCC and PAAD tissues was mainly enriched in cancer-related pathways, such as cell cycle, oocyte meiosis, and p53 signaling pathway (Fig. 4C, D). 


\section{Discussion}

In recent years, the target of cancer diagnosis and treatment has gradually shifted to cyclins [21]. SKA3 is one of the important cyclins. Although some studies have suggested that SKA3 could influence the progression of certain cancers $[22,23]$, the mechanism underlying its role is still largely unclear and controversial [24-26]. Herein, we explored the relationship between SKA3 and the diagnosis and prognosis of HCC and PAAD.

In the present study, we conducted TMA to examine the levels of SKA3 protein in PAAD and HCC. Significant decreases in SKA3 protein expression were observed in PAAD and HCC tissues relative to apposed normal tissues. This suggested that SKA3 protein could be a potential diagnostic marker for tumors. In this study, SKA3 protein expression was positively correlated with tumor size and Edmondson grade in $\mathrm{HCC}$ patients. Moreover, tumor size was a prognostic factor based on the Cox regression analysis. These results further verified that the expression level of SKA3 protein is closely related to the prognosis of HCC patients. However, in PAAD patients, we did not find factors related to the downregulation of SKA3 protein. It is worth noting that, previous studies have shown that SKA3 expression is higher in HCC tumor tissues than in para-carcinoma [27]. Hou et al. reported that SKA3 was up-regulated in HCC tissues and that downregulation of SKA3 mRNA was associated with better survival of HCC [27].

Our SKA3 protein research and GEO's RNA research are not the same. According to the GEO database, the expression level of SAK3 RNA was significantly higher in HCC and PAAD tissues. Therefore, we suspected that RNA and protein expression levels were different in the two tumors. Thus, we believe that the reason for such different results is that SKA3 may be regulated by post-translational modifications, including phosphorylation, acetylation, methylation and ubiquitination, resulting in a reduction in protein levels.

We also examined the prognostic significance of SKA3 protein in cancer prognosis. Our study showed that HCC patients with high expression of SKA3 protein had a better prognosis. In contrast, PAAD patients with a high level of SKA3 protein expression exhibited a worse prognosis. And the lower expression of SKA3 protein showed that tumor growth of HCC patients grew faster. SKA3 plays a crucial role in the establishment of stable kinetochore-microtubule interactions (end-on) and spindle checkpoint silencing $[29,30]$. As such, it speeds mitosis and shortens the cell replication cycle. However, according to our results, SKA3 proteins influenced the progression of HCC and PAAD tumors. Therefore, we speculated that SKA3 could have different biological functions in both tumors, an observation that is contrary to previous studies $[22,27]$.

We suspect that SKA3 protein has the following two biological behaviors in tumors: First, SKA3 protein promotes cell mitosis and rapid tumor growth; Second, excessive expression of SKA3 protein will inhibit cell mitosis. The latter mechanism needs further study. Hu et al. also found that overexpression of SKA3 was related to enhanced tumor growth and cell migration in cervical cancer [28]. Given the inconsistency in these results, there is a need to conduct further studies with a special focus on the role of SKA3 in the development and progression of various cancers.

Page $13 / 21$ 
However, this study had some limitations. For example, we did not conduct a qPCR quantitative analysis of mRNA content in tissues, and the pathway was not clear. Thus, more comprehensive studies are needed to verify the results of the present study.

\section{Conclusions}

Our results demonstrate that SKA3 is a promising diagnostic marker for HCC and PAAD indicator and may also act as a prognostic marker. As such, these findings can be used for clinical diagnosis and exploration of the etiology of tumors. However, the role of SKA3 in tumor development appears to be more complicated than previously anticipated and therefore requires further study.

\section{Abbreviations}

SKA3

spindle and kinetochore-related complex subunit 3; HCC:hepatocellular carcinoma; PAAD pancreatic adenocarcinoma; GEO:Gene Expression Omnibus; TMA:tissue microarray; GO:Gene ontology; KEGG:Kyoto Encyclopedia of Genes and Genomics; APC/C:anaphase-promoting complex/cyclosome body; GEPIA:gene expression profiling interactive analysis:DEGs:differentially expressed genes; IHC:immunohistochemical; BSA:bovine serum albumin; DAB:diaminobenzidine; HE:hematoxylin and eosin.

\section{Declarations}

\section{Acknowledgments}

Not Applicable.

\section{Author's Contributions}

Kuiwei Su and Yu Huang were responsible for writing the manuscript. Shufang Xie, Chen Yang and Guoqing Ru are responsible for the collection, processing and analysis of patient data. Xianglei He and Wanyuan Chen are responsible for the correction of immunohistochemical information. Hongying Pan is responsible for the detailed analysis of the data in the article using the software. Cheng Gang and Shibing Wang put forward the ideas of this article and amended the manuscript. All authors have read and agreed to the published version of the manuscript.

\section{Funding}

This work was supported by the Funds of Science Technology Department of Zhejiang Province (No. 2017C33116 to GQR, No. LGF18H160025 to XLH, No. LGF19H160027 to WYC, No. LGF18H140006 to GC), Zhejiang Provincial Natural Science Foundation of China (No. LY17H160062 to HYP).

\section{Availability of data and materials}


Not applicable.

\section{Consent for publication}

Not applicable.

\section{Declaration of Competing Interest}

The authors declare that there are no conflicts of interest.

\section{Ethics approval and consent to participate}

All patients who participated in this study signed written informed consent. This research was approved by the Ethics Committee of Zhejiang Provincial People's Hospital.

\section{Author details}

${ }^{1}$ Department of Stomatology, Bengbu Medical College, 2600 Donghai Avenue, Bengbu, 233030, China. ${ }^{2}$ Department of Stomatology, Zhejiang Provincial People's Hospital, People's Hospital of Hangzhou Medical College, Hangzhou, 310014, China. ${ }^{3}$ Academy of Chinese Medical Sciences, Zhejiang Chinese Medical University $₫$ Hangzhou $\varangle 310053 \llbracket$ China. ${ }^{4}$ The Second Clinical Medical College, Zhejiang Chinese Medical University, Hangzhou 310000, China. ${ }^{5}$ Department of Clinical Medicine, Qingdao University, Qingdao, China. ${ }^{6}$ Departments of Pathology, Zhejiang provincial people's hospital, People's Hospital of Hangzhou Medical College, Hangzhou, China. ${ }^{7}$ Department of Infectious Disease, Zhejiang provincial people's hospital, People's Hospital of Hangzhou Medical College, Hangzhou, China. ${ }^{8}$ Key Laboratory of Tumor Molecular Diagnosis and Individualized Medicine of Zhejiang Province, Zhejiang Provincial People's Hospital, Hangzhou, China. ${ }^{9}$ Molecular diagnosis laboratory, Zhejiang Provincial People's Hospital, People's Hospital of Hangzhou Medical College, Hangzhou, China.

\section{References}

1. Gaitanos Tn, Santamaria A, Jeyaprakash Aa, Wang B, Conti E, Nigg E. Stable kinetochore-microtubule interactions depend on the Ska complex and its new component Ska3/C130rf3. EMBO J. 2009;28(10):1442-52.

2. Sivakumar S, Daum T Jr, Ar, Rankin S, Gorbsky G. The spindle and kinetochore-associated (Ska) complex enhances binding of the anaphase-promoting complex/cyclosome (APC/C) to chromosomes and promotes mitotic exit. Mol Biol Cell. 2014;25(5):594-605.

3. Amin Ad P, TI, Li L, Rajan Ss, Choudhari R, Puvvada Sd, et al. Diffuse large B-cell lymphoma: can genomics improve treatment options for a curable cancer? Cold Spring Harb. Mol Case Stud. 2017;3(3):a001719.

4. Hu R, Wang Mq N, Wb W, Yy YL, Ly L, et al. SKA3 promotes cell proliferation and migration in cervical cancer by activating the PI3K/Akt signaling pathway. Cancer Cell Int. 2018;18:183. 
5. Lv T, Miao Yf, Jin K, Han S, Xu T, ZI Q, et al. Dysregulated circular RNAs in medulloblastoma regulate proliferation and growth of tumor cells via host genes. Cancer Med. 2018;7(12):6147-57.

6. Hidalgo M, Cascinu S, Kleeff J, Labianca R, Löhr Jm, Neoptolemos J, et al. Addressing the challenges of pancreatic cancer: future directions for improving outcomes. Pancreatology. 2015;15(1):8-18.

7. Van Roessel S, Kasumova Gg, Verheij J, Najarian Rm, Maggino L, De Pastena M, et al. International Validation of the Eighth Edition of the American Joint Committee on Cancer (AJCC) TNM Staging System in Patients With Resected Pancreatic Cancer. JAMA Surg. 2018;153(12):e183617.

8. Dimitrakopoulos C, Vrugt B, Flury R, Schraml P, Knippschild U, Wild P, et al. Identification and Validation of a Biomarker Signature in Patients With Resectable Pancreatic Cancer via Genome-Wide Screening for Functional Genetic Variants. JAMA Surg. 2019;154(6):e190484.

9. Resovi A, Bani Mr, Porcu L, Anastasia A, Minoli L, Allavena P, et al. Soluble stroma-related biomarkers of pancreatic cancer. EMBO Mol. Med. 2018;10(8).

10. Ferlay J, Soerjomataram I, Dikshit R, Eser S, Mathers C, Rebelo M, et al. Cancer incidence and mortality worldwide: sources, methods and major patterns in GLOBOCAN 2012. Int J Cancer. 2015;136(5):E359-86.

11. Heimbach Jk K, Lm F, Rs S, Cb A, Mm R, Lr, et al. AASLD guidelines for the treatment of hepatocellular carcinoma. Hepatology. 2018;67(1):358-80.

12. Kim Ng N, Pp, Dang H, Kumari R, Garcia G, Esquivel Co, et al. Temporal trends in disease presentation and survival of patients with hepatocellular carcinoma: A real-world experience from 1998 to 2015. Cancer. 2018;124(12):2588-98.

13. Takuma Y, Shota I, Miyatake H, Uematsu S, Okamoto R, Araki Y, et al. Nomograms to Predict the Disease-free Survival and Overall Survival after Radiofrequency Ablation for Hepatocellular Carcinoma. Intern Med. 2018;57(4):457-68.

14. Yang X, Li S, Wang H, Chen W, Mou X, Wang S. Expression of coxsackie and adenovirus receptor is correlated with inferior prognosis in liver cancer patients. Oncol Lett. 2019;17(2):2485-90.

15. Yin L, He N, Chen C, Zhang N, Lin Y, Xia Q. Identification of novel blood-based HCC-specific diagnostic biomarkers for human hepatocellular carcinoma. Artif Cells Nanomed Biotechnol. 2019;47(1):1908-16.

16. Dai X, Xue J, Hu J, Yang SI C, Gg L, Pbs, et al. Positive Expression of Programmed Death Ligand 1 in Peritumoral Liver Tissue is Associated with Poor Survival after Curative Resection of Hepatocellular Carcinoma. Transl Oncol. 2017;10(4):511-7.

17. Tang Z, Li C, Kang B, Gao G, Li C, Zhang Z. GEPIA: a web server for cancer and normal gene expression profiling and interactive analyses. Nucleic Acids Res. 2017;45:W98-102.

18. Barrett T, Wilhite Se, Ledoux P, Evangelista C, Kim If, Tomashevsky M, et al. NCBI GEO: archive for functional genomics data sets-update. Nucleic Acids Res. 2013;41:D991-5.

19. Uhlen $M$, Oksvold P, Fagerberg L, Lundberg E, Jonasson $K$, Forsberg $M$, et al. Towards a knowledgebased Human Protein Atlas. Nat Biotechnol. 2010;28(12):1248-50. 
20. Bai Xl, Zhang Q, Ye Ly, Liang F, Sun X, Chen Y, et al. Myocyte enhancer factor 2 C regulation of hepatocellular carcinoma via vascular endothelial growth factor and Wnt/ $\beta$-catenin signaling. Oncogene. 2015;34(31):4089-97.

21. Otto T, Sicinski P. Cell cycle proteins as promising targets in cancer therapy. Nat Rev Cancer. 2017;17(2):93-115.

22. Dd Hu, HI C, Lm L, Zhang H, Yang GI. SKA3 promotes lung adenocarcinoma metastasis through the EGFR-PI3K-Akt axis. Biosci. Rep. 2020;40(2).

23. Lee M, Williams K, Hu Y, Andreas J, Patel Sj, Zhang S, et al. GNL3 and SKA3 are novel prostate cancer metastasis susceptibility genes. Clin Exp Metastasis. 2015;32(8):769-82.

24. Auckland P, Clarke Ni R, Sj M, Ad. Congressing kinetochores progressively load Ska complexes to prevent force-dependent detachment. J Cell Biol. 2017;216(6):1623-39.

25. Zhang Q, Chen Y, Yang L, Liu H. Multitasking Ska in Chromosome Segregation: Its Distinct Pools Might Specify Various Functions. BioEssays. 2018;40(3).

26. Sc E-M, Sg H, Devare J, Zong H, Cai S, Carnes S, et al. Aurora B inhibits MCAK activity through a phosphoconformational switch that reduces microtubule association. Curr Biol. 2013;23(24):24919.

27. Hou Y, Wang Z, Huang S, Sun C, Zhao J, Shi J, et al. SKA3 Promotes tumor growth by regulating CDK2/P53 phosphorylation in hepatocellular carcinoma. Cell Death Dis. 2019;10(12):929.

28. Km T, Ds P, Wu Z, Han A, Triboulet R, Seligson Mt, et al. LIN28 phosphorylation by MAPK/ERK couples signalling to the post-transcriptional control of pluripotency. Nat Cell Biol. 2017;19(1):60-7.

29. Wang L, Cho Yl, Tang Y, Wang J, Park Je, Wu Y, et al. PTEN-L is a novel protein phosphatase for ubiquitin dephosphorylation to inhibit PINK1-Parkin-mediated mitophagy. Cell research. 2018;28(8):787-802.

30. Raaijmakers Ja T, Me M, Af M, Rh. RAMA1 is a novel kinetochore protein involved in kinetochoremicrotubule attachment. J Cell Sci. 2009;122:2436-45.

31. Daum W Jr, Jd D, Jj, Sivakumar S, Mcavoy Jn P, Ta, et al. Ska3 is required for spindle checkpoint silencing and the maintenance of chromosome cohesion in mitosis. Curr Biol. 2009;19(17):1467-72.

32. Raaijmakers Ja T, Me M, Af M, Rh. RAMA1 is a novel kinetochore protein involved in kinetochoremicrotubule attachment. Journal of cell science. 2009;122:2436-45.

\section{Figures}



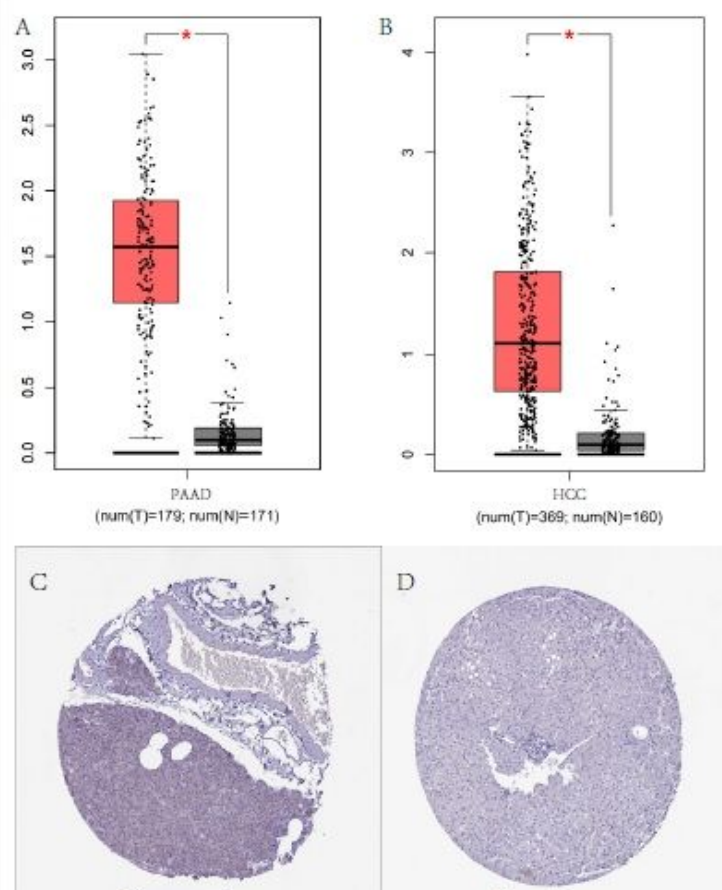

Pancreas tissue

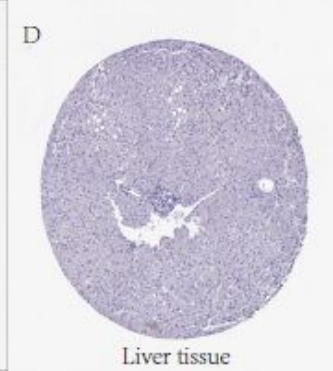

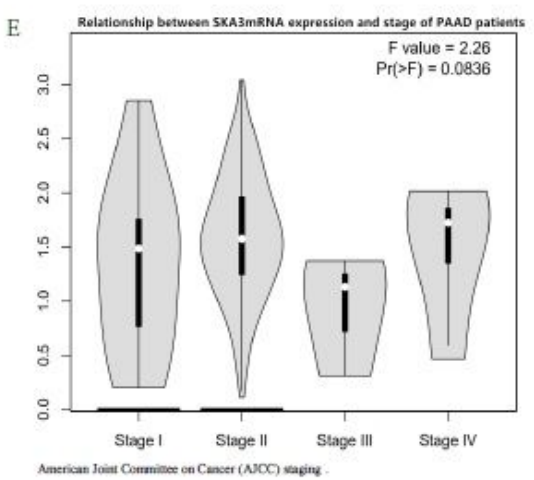
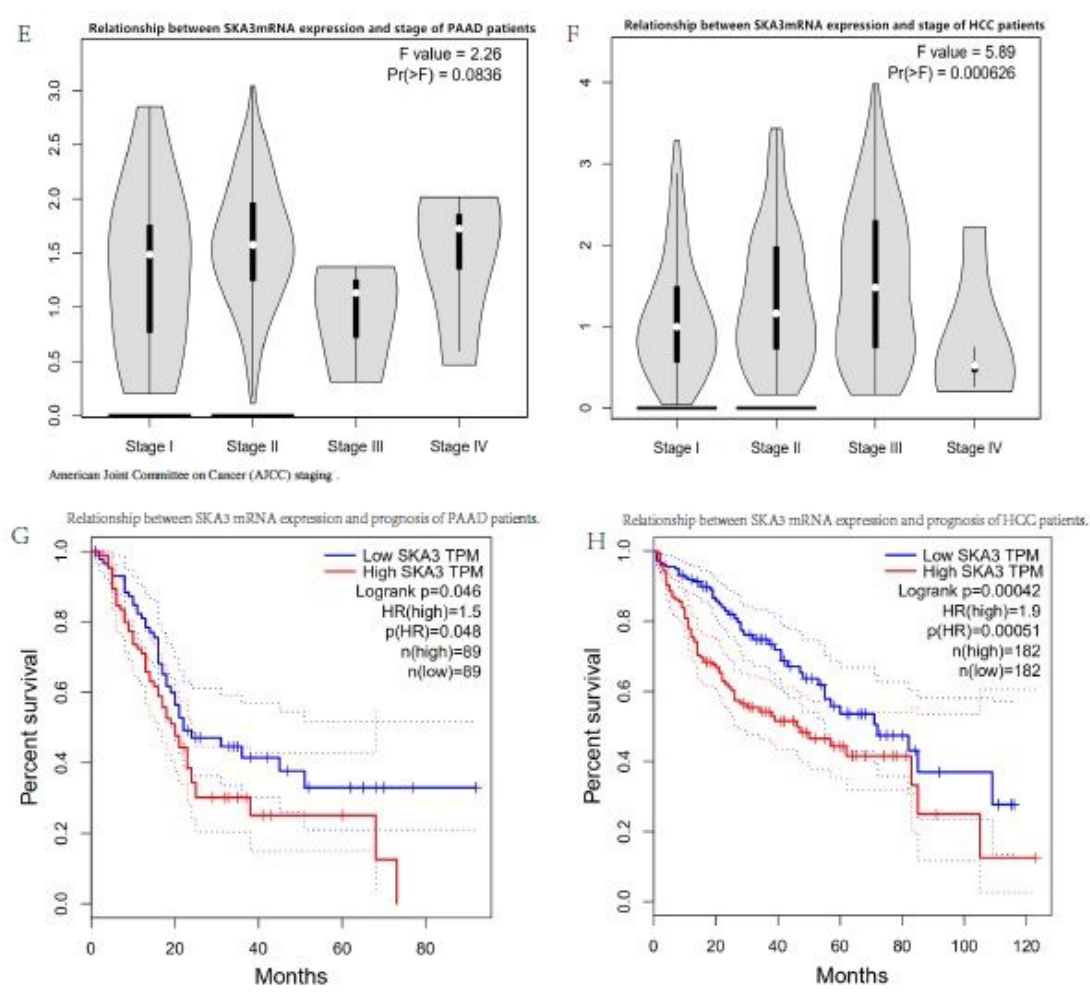

\section{Figure 1}

A and B: Differential expression of SKA3 in the liver and pancreatic cancer tissues versus normal liver tissues in the GEPIA database; $C$ and D: The human protein atlas showing that SKA3 expression was not detected in liver and pancreas tumor tissues.E and F: Differences in SKA3 mRNA expression with advanced clinical and pathological parameters in HCC and PAAD patients.GandH: Relationship between SKA3 mRNA expression and prognosis of HCC and PAAD patients. ${ }^{*} \mathrm{P}<0.05$. A P-value of less than 0.05 is statistically significant. American Joint Committee on Cancer (AJCC) staging. 

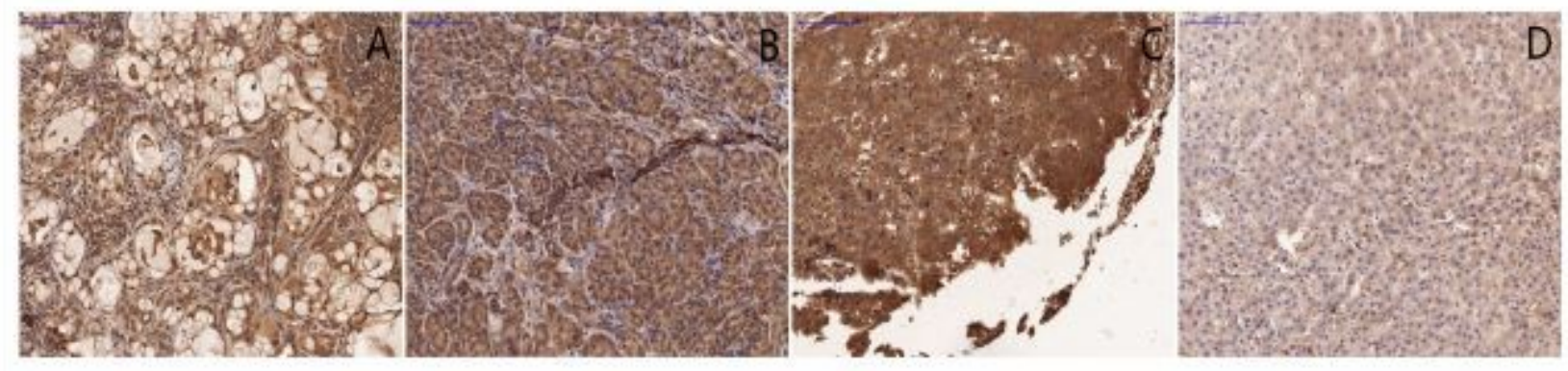

E

SKA3 was poorly expressed in HCC

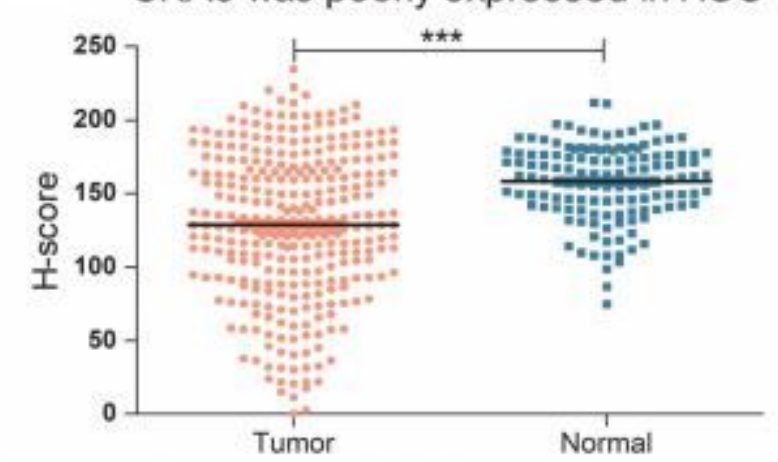

F SKA3 was poorly expressed in PAAD
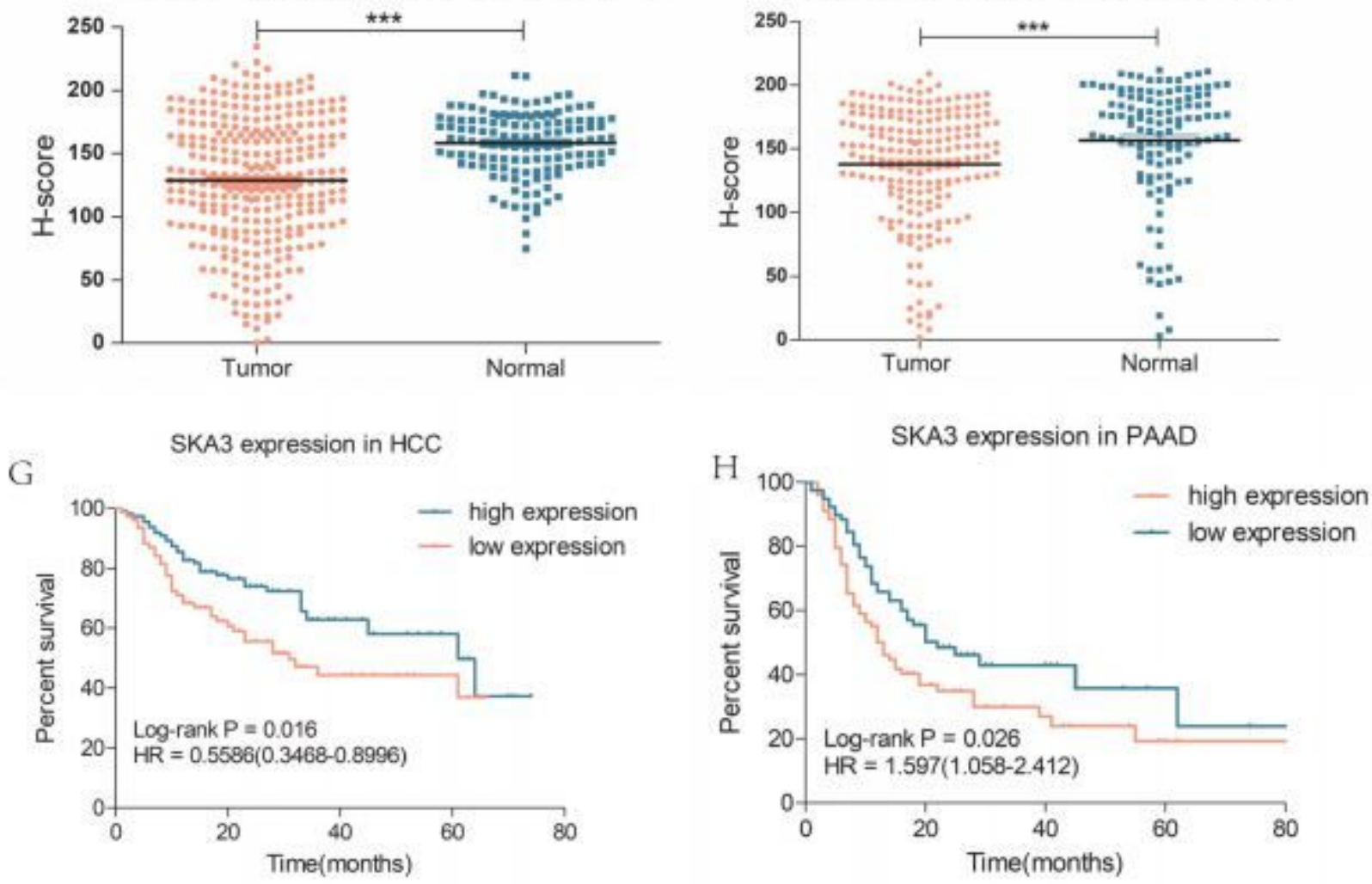

Figure 2

Differential expression of SKA3 in HCC and PAAD specimens and adjacent tissues. A: PAAD tissue; B:

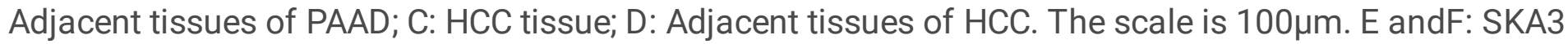
was poorly expressed in HCC and PAAD. The statistical method isthe Mann-Whitney $\mathrm{U}$ test. Confidence Intervals: 95\%. ***: $P<0.001$. Line at: Mean with SEM. G and H: Kaplan-Meier survival analysis of patients with HCC and PAAD. Overall survival curves for patients with HCC and PAAD according to the low and high expression levels of SKA3 based on IHC variables in tumor samples. Confidence Intervals: 95\%. A P-value of less than 0.05 is statistically significant. 

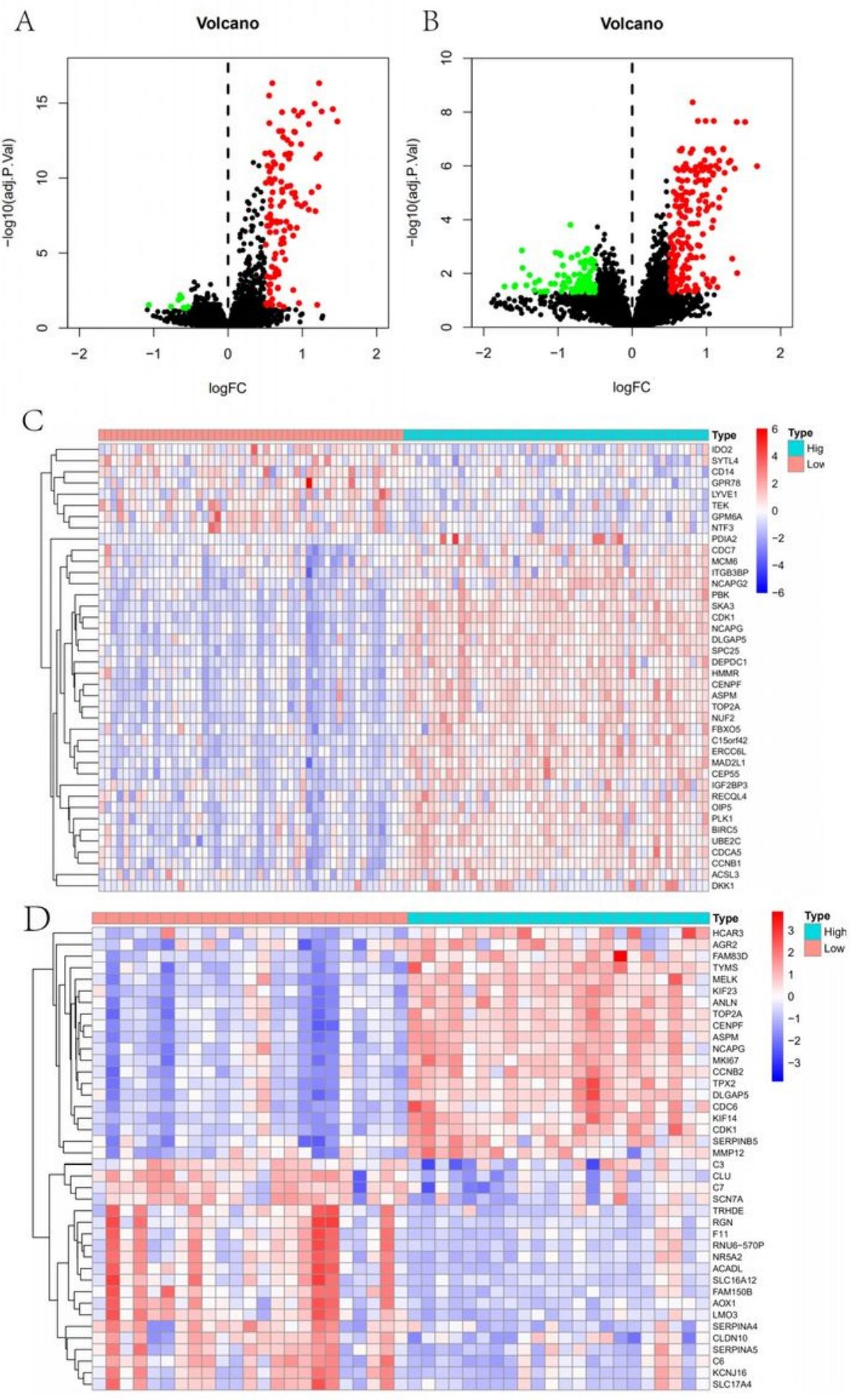

\section{Figure 3}

Enrichment analysis between DEGs. A and B: Volcano diagrams show the distribution of DEG in up- and down-regulated genes. Green represents down-regulated genes, whereas red represents up-regulated genes. A: HCC tissue; B: PAAD tissue. 
A
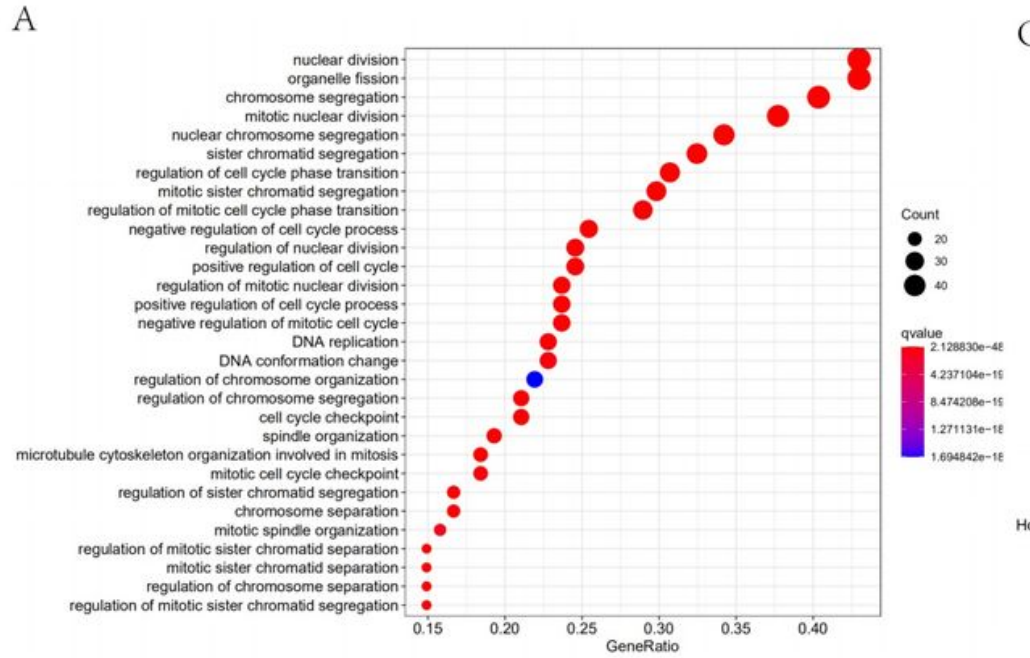

B

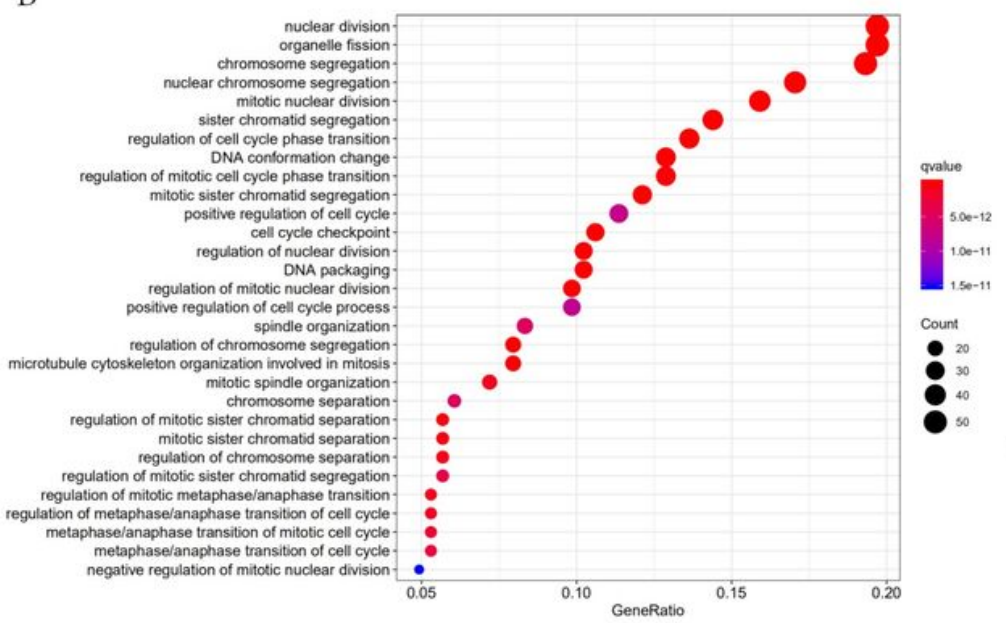

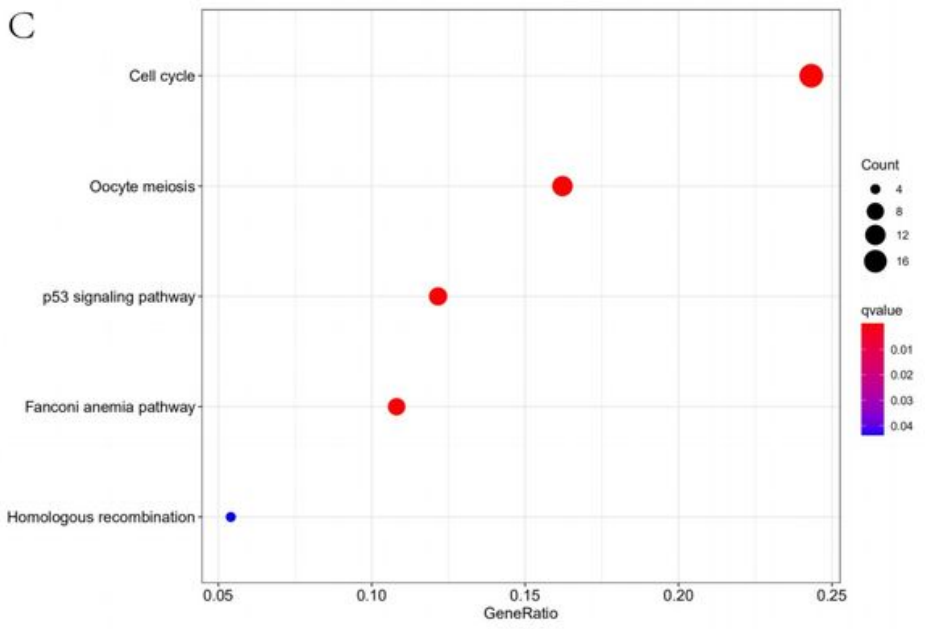

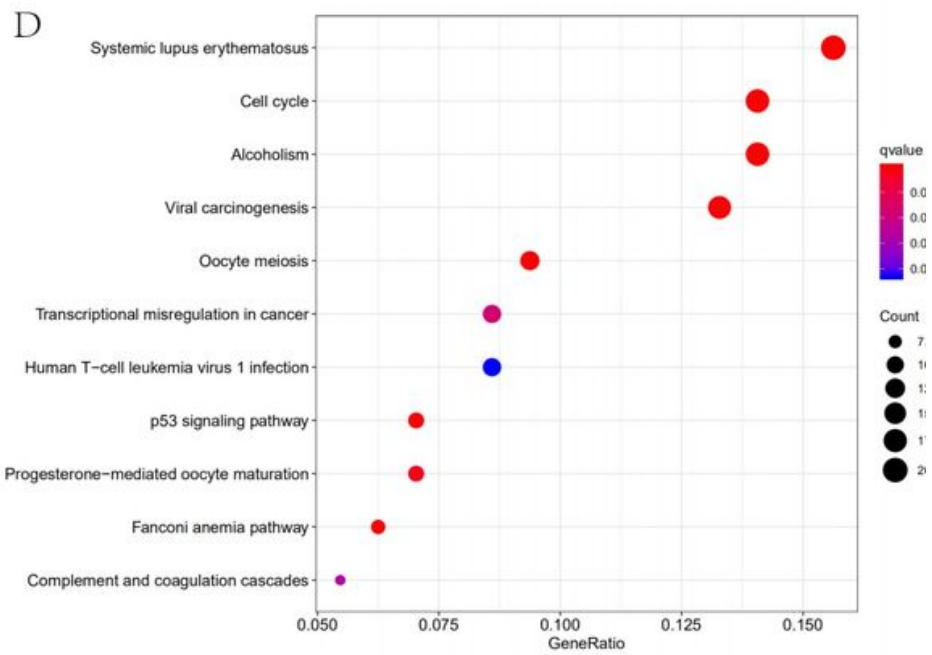

\section{Figure 4}

A andB:Top enriched GO terms for the turquoise module. C andD:The enriched KEGG pathways for the turquoise module. A and C: GSE62044 (HCC); B and D: GSE28735 (PAAD). 